\title{
Surgical Management of Complex Middle Cerebral Artery Aneurysms
}

\author{
Fernando G. DiAZ, Murali GUTHIKONDA, Lisa GUYOT, Bernard VELARDO*, \\ and Vickie GORDON
}

\author{
Neurological Surgery Department and *Anesthesia Department, Wayne State University \\ School of Medicine, Detroit, Michigan, U.S.A.
}

\begin{abstract}
Complex middle cerebral artery (MCA) aneurysms are defined in this review as aneurysms larger than $20 \mathrm{~mm}$, arising from the MCA bifurcation, and requiring unusual surgical approaches for their obliteration. The direct surgical approaches to complex MCA aneurysms can be divided into five techniques: 1) direct clipping, 2) trapping, 3) trapping and extracranial-intracranial anastomosis, 4) excision and endto-end anastomosis, and 5) external wrapping. The pertinent surgical anatomy, preoperative preparation, intraoperative procedures, operative approaches, and potential complications will be reviewed.
\end{abstract}

Key words: complex middle cerebral artery aneurysms, surgical approaches

\section{Introduction}

Aneurysms arising from the middle cerebral artery (MCA) represent $33 \%$ of all intracranial aneurysms. ${ }^{20}$ The intimate relationship of the MCA and its branches with the sylvian fissure, and the distribution of the MCA flow, make these aneurysms challenging, and potentially complicated in their surgical management. Great attention to detail and clear understanding of the operative anatomy of the MCA, facilitate the safe and successful treatment of these aneurysms. Complex MCA aneurysms are defined in this review as aneurysms larger than $20 \mathrm{~mm}$, arising from the MCA bifurcation, and requiring unusual surgical approaches for their obliteration. The direct surgical approaches to complex MCA aneurysms can be divided into five techniques: 1) direct clipping, 2) trapping, 3) trapping and extracranial-intracranial anastomosis, 4) excision and end-to-end anastomosis, and 5) external wrapping. The pertinent surgical anatomy, preoperative preparation, intraoperative procedures, operative approaches, and potential complications will be reviewed. Emphasis is placed on future methods to be used to prevent or limit the potential complications experienced in some of these patients.

\section{Clinical Material}

The surgical experience with 750 anterior circula- tion aneurysms is presented. Aneurysms arose from the internal carotid artery: 345 (46\%), MCA: 193 $(26 \%)$, and anterior cerebral artery: $212(28 \%)$. There were $100(13 \%)$ giant aneurysms. Complex MCA aneurysms are defined in this review as aneurysms larger than $20 \mathrm{~mm}$, arising from the MCA bifurcation, and requiring unusual technical approaches for their obliteration. There were 48 complex MCA aneurysms, measuring $20-84 \mathrm{~mm}$ in maximum diameter, presenting in patients of ages ranging from 23 to 72 . Fifteen patients presented with subarachnoid hemorrhage; 17 had focal neurological findings secondary to mass effect, and 16 had chronic headaches as the presenting complaint. Headaches, however, were found in all patients without exception. Patients who had a subarachnoid hemorrhage were admitted to an intensive care unit for evaluation, in preparation for surgery, ${ }^{27]}$ others were admitted electively for surgery. A complete preoperative medical evaluation was obtained in elderly patients to prevent intraoperative cardiac, respiratory, or renal complications that may develop from the use of mannitol or cardiac suppressants such as barbiturates or lidocaine, or from hypertension, hypotension, or blood loss. ${ }^{27]}$ A Swan-Ganz catheter, an intra-arterial recording catheter, and a Foley catheter were inserted in preparation for surgery. All patients received dexamethasone $10 \mathrm{mg}$ intravenously on admission and $4 \mathrm{mg}$ every 6 hours, and nimodipine $1 \mathrm{mg} / \mathrm{kg}$ P.O. until surgery. Salt 
poor albumin was infused until a cardiac output of at least $5-7 \mathrm{l} / \mathrm{min}$ was obtained.

Anesthesia was induced with intravenous thiopental and a non-depolarizing muscle relaxant. Complete muscle relaxation with hemodynamic stability can be achieved using vecuronium cis-atracurium. Anesthetic induction adjuvants include lidocaine, fentanyl, and esmolol. To maintain the patient in a stable hemodynamic condition is necessary to have stable cardiac filling pressures with the administration of volume expanders, and the maintenance of normal $\mathrm{PaO}_{2}$, provided by mask ventilation prior to laryngoscopy and intubation. Arterial blood gases, end expiratory $\mathrm{CO}_{2}$ recordings, and pulse oximetry are monitored throughout the operation. $\mathrm{PaCO}_{2}$ is maintained at about $30-35 \mathrm{mmHg}, \mathrm{PO}_{2}$ at about 95 $\mathrm{mmHg}$, and oxygen saturation at about $100 \%$. The patients are maintained normothermic and normotensive throughout the operation. Intraoperative hypotension is only used when proximal arterial control cannot be accomplished with a temporary clip.

During induction, the patients received intravenous diphenylhydantoin $15 \mathrm{mg} / \mathrm{kg}$, vancomycin $1000 \mathrm{mg}$, dexamethasone $10 \mathrm{mg}$, gentamycin 80 $\mathrm{mg}$, furosemide $20-40 \mathrm{mg}$, and mannitol $0.5 \mathrm{~g} / \mathrm{kg}$. Spinal fluid drains were not used, since drainage of spinal fluid from the cisterns and the lamina terminalis was effective in achieving cerebral relaxation. Arterial blood gases, end expiratory $\mathrm{CO}_{2}$ recordings, and pulse oximetry were monitored throughout the operation.

Twenty-nine patients underwent direct clipping of the aneurysm, five patients had the aneurysm trapped, and five others had an extracranial-intracranial anastomosis in addition to trapping. The MCA was anastomosed directly after the resection of the aneurysm in three patients, and two others had an end-to-side anastomosis of adjacent MCA branches. One patient had wrapping of the aneurysm with muslin. Temporary clamp times varied from 7 to 155 minutes; eight patients did not have any temporary clipping. The aneurysm had to be opened and decompressed in 22 patients, with removal of formed thrombus in all, and removal of wall atheroma in 10 patients. Immediate neurological worsening was noted in 17 patients, 22 remained unchanged from preoperative status, three improved, and six patients died in the initial postoperative period. Four mortalities occurred in patients who presented with a severe subarachnoid hemorrhage (Hunt and Hess ${ }^{17)}$ grades 4 and 5, Fischer grade 4), and developed vasospasm in the immediate postoperative period. Two patients died from cerebral infarction; in one it was not possible to reconstruct more than one major trunk of his MCA, and in the other the only explanation was a clamp time of 72 minutes with a normal angiographically appearing MCA territory. One more patient died from an intracerebral hematoma in the immediate postoperative period.

Immediate postoperative angiography was performed in 43 patients within 48 hours of surgery. Angiograms were not done in four patients who died in the immediate postoperative period, and in one patient who had an excellent postoperative result. The angiograms revealed the complete obliteration or exclusion of the aneurysm in every patient. All extracranial-intracranial anastomoses were patent, and all intracranial reconstructions including endto-side and end-to-end anastomoses were patent. Collateral flow was observed in the territories of the distal arteries which were trapped and not bypassed. Two of four patients who died had severe arterial vasospasm. In another patient who died, only one branch of an aneurysm measuring $66 \mathrm{~mm}$ could be reconstructed and flow through the vessel was insufficient to supply the entire MCA distribution. One patient revealed complete occlusion of the MCA at the level of the clip.

Long-term recovery at 5 years (12-80 months) was excellent. Thirty-nine patients returned to full functional recovery, one patient was moderately impaired, and two patients had a severe neurological deficit. These last two patients had their deficits preoperatively, and they remained unchanged after surgery. One patient who developed a cerebral infarction immediately after surgery died in the longterm follow-up. Initial worsening of neurological deficits in the immediate postoperative period, as well as preoperative deficits, improve sufficiently on long-term follow-up to allow these patients to resume independent living. All surviving patients returned to independent living, although only eight were able to return to work.

\section{Discussion}

\section{Anatomical considerations}

The internal carotid artery becomes subarachnoid as it arises from the cavernous sinus. From the cavernous sinus to the bifurcation, the internal carotid artery is variable in length, and in the number of branches it gives rise to. Besides the ophthalmic artery, the posterior communicating and anterior choroidal arteries, there are branches directed to the anterior perforated substance, and in some cases, a proximal branch to the temporal pole or to the inferior frontal area. ${ }^{12-15)}$ The perforating branches generally arise from the posterior wall of the internal carotid artery, either before or after the bifurca- 
tion, not from the bifurcation itself. The perforating branches from the anterior cerebral artery may arise from a common trunk, or they may arise as several small branches directed to the medial portion of the anterior perforated substance. ${ }^{13,14)}$ The anterior perforating arteries originating from the MCA arise from a single common trunk or from several small perforating branches. $^{25,26)}$ These MCA perforators may originate from the most medial portion of the artery, close to its origin $(37 \%)$, or they may originate from the middle one third of the $M_{1}$ segment ( $47 \%$ ). The remaining (16\%) perforating vessels arise from the most lateral portion of $\mathrm{M}_{1}$.

The pattern of arterial supply to the anterior perforated substance is one of dominance by either the anterior cerebral artery or the MCA; the branches arising from the other artery will be smaller and fewer in number. ${ }^{14,15,25)}$ In a few cases a large vessel may arise from the distal portion of the $A_{1}$ segment and go to the anterior perforated substance retrogradely, substituting for the recurrent artery of Heubner. When the territories of the anterior cerebral artery and MCA perforators overlap, permanent occlusion of one perforator may not be of great importance, and may go unnoticed clinically. However, when the perforating vessel is dominant, permanent occlusion of this artery may be followed by severe and lasting neurological deficit.

As the anterior cerebral artery passes from the carotid bifurcation to the interhemispheric fissure, it gives rise to branches to the anterior perforated substance, the inferior frontal area, the frontopolar artery, the recurrent artery of Heubner, the septal perforating arteries, and the anterior communicating artery. The anterior cerebral artery then continues in the interhemispheric fissure towards the convexity. ${ }^{12.13,29)}$ The recurrent artery of Heubner and the septal perforators are the most important branches of the proximal portion of the anterior cerebral artery, supplying the anterior limb of the internal capsule, the anterior commissure, the septal region, the anterior portion of the fornix and hypothalamus, and the most anterior portion of the thalamus. ${ }^{1213.29)}$ The MCA continues in the sylvian fissure and divides in two or three primary trunks, from which will originate the branches to the cerebral cortex. ${ }^{25,261}$ Particularly important are the branches to the Rolandic area, the angular gyrus, and the opercular area. Damage to one of these branches is poorly tolerated. Every branch of the MCA should be identified prior to permanent clip placement. ${ }^{24)}$ The sylvian veins are predominantly located on the temporal side of the sylvian fissure and can be easily identified and spared. In most patients, the temporal tip veins may be coagulated and tran- sected, but in some cases damage to these veins may result in venous congestion, cerebral edema, or venous infarction. ${ }^{28)}$

Yasargil et al. ${ }^{281}$ described the subarachnoid cisterns at the base of the brain as small envelopes which encircle all structures contained in the subarachnoid space. In the supraclinoid carotid area, there are cisterns about the internal carotid artery, the carotid bifurcation, the MCA, the anterior cerebral artery, the optic nerve, the chiasm, the prechiasmatic cistern, the cistern of the lamina terminalis, and the confluence of the sylvian fissure to the middle cerebral cistern. Wide opening and sharp dissection of all cisterns is required to expose complex MCA aneurysms safely. ${ }^{11)}$

\section{Diagnostic evaluation}

Computed tomography and magnetic resonance imaging are necessary to exclude intra- or extraparenchymal hematomas, the extent of the subarachnoid hemorrhage, the presence of hydrocephalus, and the possibility of intra-aneurysmal clot formation. ${ }^{2,8,18)} \mathrm{A}$ false sense of security may be gained from an angiogram which reveals an apparently small aneurysm, when in reality the aneurysm is large, and filled with clot. ${ }^{1,2,5,16)}$ Magnetic resonance imaging facilitates the localization of the hemorrhage within the parenchyma of the brain, and may suggest the presence of a vascular abnormality. A selective four vessel cerebral angiogram must be completed in all patients suspected of having an aneurysm, to determine its location, to exclude the presence of multiple aneurysms which occur in $20 \%$ of cases, and to rule out the presence of vasospasm. ${ }^{1,2,8,18 \text { ] }}$

\section{Preoperative preparation}

Careful monitoring of the arterial blood pressure with an intra-arterial line will facilitate the management of the blood pressure, and prevent undesirable fluctuations. Fluid balance and cardiac function are best managed with a Swan-Ganz catheter. An optimum intravascular blood volume may be established preoperatively by the administration of intravenous albumin, and the careful monitoring of the cardiac output and cardiac index. Patients with chronic obstructive pulmonary disease or with previous pulmonary problems may require securing arterial blood gases or pulmonary function tests, in addition to a chest $\mathrm{x}$-ray. ${ }^{27)}$

Brain protective drugs such as diphenylhydantoin, dexamethasone, and calcium channel blockers may be administered in preparation for surgery. ${ }^{3-5,7,11,24)}$ Suzuki $^{24 l}$ observed that diphenylhydantoin has a brain protective effect during temporary clipping, 
exerted by stabilizing the neuronal membrane and reducing membrane excitability. Dexamethasone has been studied extensively as a possible cerebral protective agent, and found effective only when given prior to the occlusion of a cerebral artery. Since the effect of diphenylhydantoin and dexamethasone can be maximized by early administration and tissue saturation, they are ideal for preoperative administration.

Calcium channel blockers reduce the incidence of cerebral ischemic complications associated with arterial vasospasm, when administered in the immediate post-hemorrhagic period. ${ }^{18)}$ Their effect results from a direct vasodilator action on the smooth muscle cell of the cerebral vessels. In addition, calcium channel blockers may have a direct protective effect at a molecular level in the ischemic neuron, by preventing the sudden influx of calcium, and blocking the onset of the ischemic cascade triggered by the intracellular accumulation of calcium. Calcium channel blockers are given from the time of admission until after surgery. Aminocaproic acid has fallen in disfavor and is no longer recommended, because of the numerous ischemic complications and the increased incidence of hydrocephalus observed with its use. ${ }^{4)}$

The decision to proceed with surgery should be based on the clinical condition of the patient, including the neurological evaluation and the general medical status. Patients with poor medical condition but with good neurological status should not be operated on any sooner than the patient with poor neurological condition but a favorable medical state. ${ }^{27)} \mathrm{A}$ poor condition in either case would likely lead to a poor outcome. In general, most would agree that patients with a Hunt and Hess grade of three or lower may be operated on almost any time.$^{4,11,17,24)}$ In the presence of vasospasm, most would tend to wait until vasospasm has lapsed, although patients with a good neurological condition but with angiographic arterial narrowing may do well with surgery. ${ }^{3,4]}$

\section{Transoperative management}

The preparation prior to the induction of anesthesia for patients with intracranial aneurysms requires meticulous care. Comprehensive care mandates the clinical assessment of central nervous system function, the placement of non-invasive monitoring, and establishing peripheral venous access in a calm, controlled environment. The use of local anesthetic for placement of invasive monitoring devices is essential. Preoperative changes in central nervous system function requires a thorough investigation to prevent the possible rupture of the aneurysm, or the development of hypoxemia or hypotension. The anxious patient may tolerate judicious use of intravenous sedation. Placement of an arterial line and a pulmonary artery catheter for measurement of central venous pressure, pulmonary wedge pressure, cardiac output, and cardiac index facilitate the management of the surgical procedure. ${ }^{271}$

Patients with MCA aneurysms require a mild state of hyperventilation to keep the $\mathrm{PCO}_{2}$ at about 30 to $35 \mathrm{mmHg}$. Lowering the $\mathrm{PCO}_{2}$ below $30 \mathrm{mmHg}$ will not aid further in reducing cerebral volume, and may in fact induce cerebral ischemia, by promoting vasoconstriction. Hyperventilation-induced vasoconstriction is particularly a problem for patients with residual vasospasm who are already in a partial ischemic state. ${ }^{271}$ Hyperventilation-induced hypocapnia during the period of temporary clipping is not recommended because it has the potential of worsening the transocclusion ischemia, and may potentiate the neurological deficits.

Mannitol is used to decrease brain volume during surgery, and to facilitate brain retraction. Suzuki ${ }^{24)}$ reported a beneficial effect in brain tolerance to cerebral ischemia, when mannitol was given during surgery. The benefit of mannitol may result from its action as an anti-aggregant, enhancing microcirculatory flow, permitting greater corpuscular deformation, and allowing greater release of oxygen at tissue levels. More recent evidence indicates that mannitol also has a free radical quenching ability. Thiopental acts as a cerebral protective agent producing a functional reduction of oxygen consumption at the synaptic junction, estimated at $80 \%$ of the metabolic activity of the brain. ${ }^{7,11,22,24,271}$ This protective effect is demonstrable when the thiopental levels are sufficient to produce an isoelectrical tracing on electroencephalography. The metabolic action of thiopental may be enhanced by the administration of lidocaine, since lidocaine blocks directly the sodium-potassium pump. ${ }^{27)}$ The intraoperative administration of barbiturates and lidocaine in preparation for temporary clipping may produce immediate hypotension and lead to myocardial ischemia.

\section{Surgical approaches}

Multiple surgical approaches have been reported to manage internal carotid artery aneurysms since Dandy ${ }^{97}$ treated the first patient with a correctly diagnosed intracranial aneurysm. Various surgical clips have permitted the obliteration of small and giant aneurysms, facilitated by intracranial vascular reconstructive and endovascular techniques. The preferable route of approach to complex MCA aneurysms is via the pterional exposure, through which direct surgical approaches to complex MCA aneurysms can be divided into five techniques: 1) 
direct clipping, 2) trapping, 3) trapping and extracranial-intracranial anastomosis, 4) excision and end-to-end anastomosis, and 5) external wrapping.

Pterional approach: A detailed description of the patient's position, the craniotomy and dissection of the anterior portion of the circle of Willis, and the clipping techniques will be reviewed. ${ }^{28} \mathrm{~A}$ description of the principles of temporary clipping, aneurysmal opening, debulking, resection, and reconstructive techniques will complete this section.

The patient's position is critical in the success of the procedure. The patient is placed supine, in a reclining position, supported by skeletal fixation, with the head rotated $15^{\circ}$ to the opposite side and with $15^{\circ}$ of extension. In this position, the sphenoid wing is perpendicular to the floor. Yasargil et al. ${ }^{28)}$ recommended the head be placed with the nose turned $30^{\circ}$ towards the opposite side, and the malar eminence uppermost on the head. When the head is in this position, the temporal lobe is difficult to dissect and the axis of the sylvian fissure rotates away from the surgeon. An incision is made along the hairline from the zygoma to the ipsilateral mid pupillary line. The skin and muscle are reflected in one single flap, exposing the entire squamosal portion of the temporal bone, the frontalis process of the lateral orbital wall, and the vertical portion of the frontal zygomatic area. The posterior branch of the superficial temporal artery is dissected and preserved as the flap is developed. Yasargil et al. ${ }^{28)}$ recommended a complex muscle incision disinserting the temporalis muscle from its anterior attachment, and retracting it posteriorly, after separating the fatty tissue which contains the frontalis branch of the facial nerve. However, this dissection lengthens the operation, and does not protect the nerve any more than elevation of a single flap.

A free bone flap is elevated, centering the craniotomy on the pterion. The pterion is drilled down until the meningo-orbital branch of the middle meningeal artery is exposed. The dura is sutured to the skull, opened in a semicircular manner based on the pterion, and sutured to the temporalis muscle. The sylvian fissure is opened under the microscope from the surface down. Extreme care must be paid to the direction of the aneurysm dome in patients with large or giant MCA aneurysms, since the dome may be entered prematurely, and extensive bleeding may ensue when the proximal MCA has not been exposed first for control.

Proximal control is achieved by dissecting the internal carotid artery as it arises from the supraclinoid area, next to the optic nerve. Once proximal control is accomplished, the rest of the sylvian fissure may be opened widely. All arachnoidal bands are released with sharp dissection, to expose the entire course of the internal carotid artery and its bifurcation, the optic nerve and optic chiasm, the proximal portion of the anterior cerebral artery, the trunk and bifurcation of the MCA to the level of the limen insula. The sylvian fissure is then relaxed, and the frontal and temporal lobes fall apart without traction. The temporal lobe is supported by the sphenoid wing and the sylvian veins, and generally it is only necessary to use a single retractor on the frontal lobe. ${ }^{19)}$ A temporal retractor is generally not needed in small MCA aneurysms, but is usually required in large or giant aneurysms.

Spinal fluid is drained gradually from the cisterns to obtain maximum brain relaxation with minimal brain retraction. Opening the sylvian fissure is facilitated by not using a lumbar drain; since the subarachnoid space is full, the sylvian fissure is readily apparent, and the dissection can be done without difficulty. When it is necessary to remove additional spinal fluid, it may be obtained opening the lamina terminalis above the chiasm. Opening the lamina terminalis is easy to do once all cisterns are open, and does not carry any of the complications of ventricular puncture. ${ }^{19)}$ Incomplete opening of the arachnoid cisterns leads to tension on the frontal and temporal lobes, requires increased pressure on the retractor blades, and provides less exposure of the area. Incomplete dissection of the cisterns may also lead to premature rupture of the aneurysm, from traction on the brain. Preservation of the veins is much easier when the cisterns have been dissected widely. Since there is less retractor pressure on the brain, the possibility of venous tears, venous stasis, or venous infarcts is reduced. The actual placement of the clip on the aneurysm neck is much easier when the area of exposure is wide.

Direct surgical approaches: Direct surgical clipping of the neck of the aneurysm remains the only truly curative approach in the treatment of intracranial aneurysms. ${ }^{23}$ Direct clipping requires complete dissection of the neck of the aneurysm, identification of all afferent and efferent vessels, dissection and isolation of important perforating arteries, and application of the clip on the neck of the aneurysm, flush with the parent vessel. Care must be taken not to cause kinks or stenosis of the parent vessel. Positioning the clip across the axis of the vessel sometimes leaves small areas of the neck outside the clip. For patients with a recent hemorrhage or patients with a large or giant aneurysm, it is safer to apply temporary clips while dissecting the aneurysm neck free from the perforators. Temporary clips may be applied to any intracranial vessel for short periods of time, which vary with the adminis- 
tration of brain protective agents. ${ }^{\text {.) }}$

When the aneurysm is large or giant, it will be necessary to use temporary clips, and deflate the aneurysm before applying the permanent clip. The aneurysm may be deflated by needle aspiration or puncturing the dome. Once the sac is collapsed, all important arterial branches and perforators may be identified and dissected, and the permanent clip may be placed safely across the neck of the aneurysm. Care must be taken not to encroach on the parent vessel, since the actual diameter of the parent vessel is also decreased by the absence of blood. In some giant aneurysms, it is not possible to clip the neck because the aneurysm is filled with thrombus or is arteriosclerotic. In aneurysms full of thrombus, it is generally safe to isolate the aneurysm complex as described, apply temporary clips, and then open the aneurysmal dome with a knife. An ultrasonic aspirator is then introduced to remove the thrombus, leaving only the supple aneurysm sac behind. The perforators can then be separated away from the aneurysm neck, and a clip applied flush with the parent vessel. It is generally not recommended to remove the aneurysmal sac, since this can be followed by unnecessary injury to the perforators and other adjacent structures.

When the aneurysmal wall is infiltrated with atheroma, it is sometimes possible to perform an endarterectomy of the aneurysmal wall, preserving the aneurysmal wall itself with a small amount of atheroma. In some cases, this may be done with ease, but it requires extreme patience. When the atheroma cannot be completely resected, a hemostatic clamp may be used to crush the atheroma and develop a smooth surface over which the clip may then be applied. When neither of these maneuvers are successful, it becomes necessary to either resect the aneurysm at the neck and perform an aneurysmorrhaphy, or to clip the aneurysm neck to include a major branch, and then perform an extracranial-intracranial anastomosis, distal to the clip.

An alternative to temporary clipping and complete obliteration of the aneurysmal neck was the use of aneurysm glues with or without wrapping. ${ }^{21)}$ Wrapping and gluing techniques failed because they required that the entire aneurysm complex be included in the encasement. In most cases, the inability to expose the entire complex is what motivated the use of these techniques as substitutes. In addition, none of the glues adhere to the vessel wall, leaving a virtual space between the glue and the aneurysm wall, where the aneurysms would then expand and rebleed. Cotton is the only substance that has in fact produced a strong enough arachnoidal reaction to reinforce the aneurysm. Gluing and wrapping have largely been abandoned.

Complex aneurysms of the MCA bifurcations represent a serious problem when one of the arterial trunks arises directly from the waist of the aneurysm. Clipping the aneurysm neck to include the origin of an arterial branch may be tolerated in a few individuals when the collateral arteries are large. However, when a major arterial trunk is included in the clip, it may be necessary to first perform an extracranial-intracranial anastomosis distal to the vessel. ${ }^{5,6.11)}$ One could rely on collateral circulation to take over the territory of the occluded vessel, but in most, the collateral circulation is insufficient when it has to come over the cerebral convexity. The extracranial-intracranial anastomosis may be performed on the surface of the hemisphere, but it is preferable to perform the bypass as close as possible to the area of occlusion. ${ }^{10,11)}$

Some giant aneurysms may not be clipped because of extensive atheroma within their wall, or because the branches which arise from the aneurysm dome are too numerous. In some patients, it is possible to resect the entire aneurysm and reconstruct the various branches. ${ }^{5,6\}}$ The principles involved in reconstruction of the anterior circulation branches include a wide dissection of the cisterns, careful isolation and identification of the afferent and efferent branches to the aneurysm, isolation and preservation of the perforators, and the use of temporary clipping with cerebral protective agents. Having isolated all branches, one must consider initially performing an extracranial-intracranial anastomosis to the largest branch arising from the aneurysm in preparation for the period of prolonged cerebral ischemia. ${ }^{101}$ In some patients, it is then possible to resect the branch or branches arising from the aneurysm, and to perform an end-to-side anastomosis to the artery with the extracranial-intracranial anastomosis or to the branch that will remain attached to the parent vessel. It may be necessary to resect the entire aneurysm, and then perform an end-to-end anastomosis of the parent vessel.

Silverberg et al. ${ }^{221}$ recommended cardiac arrest in combination with deep hypothermia when dealing with giant aneurysms. Achieving $24^{\circ} \mathrm{C}$ of core cooling, the brain will be protected for one hour of complete circulatory arrest. Draining the cerebral circulation allows the aneurysm to collapse, and permits the surgeon to dissect the perforators and branches away from the aneurysm, and allows placement of the clip on a supple aneurysm sac. Draining the blood from the cerebral circulation also empties blood from the smaller vessels, and makes their identification difficult. In addition, one must deal with the problems of full heparinization and immediate 
bleeding once the cardiac bypass is restarted and the circulation begins.

\section{Long-term results}

New postoperative deficits developed immediately after surgery in 17 patients, and all but three returned to better than preoperative levels in longterm follow-up (12-80 months). Two of these patients had a permanent neurological deficit resulting from incomplete reconstitution of the branches of the MCA distal to the trapping procedure, one patient died on long-term follow-up, and the other one, a grade 4 subarachnoid hemorrhage, survived with a deficit. The remaining patient who deteriorated had a clamp time of 60 minutes. Three of the patients who deteriorated had a trapping procedure without a distal extracranial-intracranial anastomosis. Clamp time on 14 of the patients who deteriorated immediately after surgery, but who recovered without residual varied from 0 to 67 minutes.

It is possible that the immediate postoperative deficits resulted from transoperative ischemia. However, the duration of clamp time did not correlate with the presence of postoperative deficits. Two patients who did not have any temporary clamp time may have developed a deficit from distal embolization from the aneurysm wall. Three of four patients who had a trapping procedure without a distal extracranial-intracranial anastomosis developed postoperative deficits, which eventually recovered to better than preoperative levels. Postoperative angiography revealed distal flow into the isolated branches through leptomeningeal collaterals.

Four of the seven patients who died had severe postoperative arterial narrowing and no filling of the distal MCA territory past the level of the aneurysm clipping, following a grade 4 or 5 subarachnoid hemorrhage and probably should not have been subjected to surgery. Another patient with a giant aneurysm measuring $66 \mathrm{~mm}$ died when it was not possible to reconstruct two out of three distal MCA branches. Two other patients died without a preceding subarachnoid hemorrhage, one from an intracerebral hematoma and the other from a cerebral infarction. The latter patient died on longterm follow-up after an immediate postoperative infarction and a temporary clamp time of 72 minutes.

\section{Conclusions}

The neurological deficits and mortality other than that related to vasospasm following subarachnoid hemorrhage can be attributed to incomplete restoration of the MCA territory during the operative procedure or to distal embolization at the time of surgery.
One patient died and two others had transient worsening of their neurological condition immediately postoperatively as a result of the proximal occlusion of the MCA flow, without adequate immediate reconstruction beyond the level of the occlusion. Fourteen patients who had transient worsening of their deficits immediately after direct clipping of their aneurysms had complete reconstitution of their MCA territory distal to the clip, and had angiographic preservation of all the branches arising from the MCA area. It is therefore more likely that these patients who developed a transient deficit and had a temporary clamp time under 30 minutes must have another source for their deficit, such as an embolus arising from the aneurysm or occlusion of a lenticulostriate artery. Since the patients recovered relatively rapidly in the postoperative period, and the angiograms did not reveal the loss of any lenticulostriate branches, it is not likely that these arteries were occluded, but is more probable that the deficits were the result of embolic material arising from the aneurysm.

There are only two patients in the present series whose deficits are possibly ascribed to prolonged temporary clipping. One patient had an occlusion time of 72 minutes, and died after a cerebral infarction without an angiogram. The other patient had a direct clipping of the aneurysm with a temporary occlusion time of 60 minutes. From these two patients it could be concluded that prolonged temporary occlusion beyond 60 minutes cannot be tolerated and should be discouraged. However, five other patients in this series had clamp times of $60,67,84,87$, and 155 minutes, respectively, which were well tolerated and were followed by an excellent postoperative recovery. It could be argued that the level of tolerance of specific patients will be different among individuals in a given population, and that the surgeon may not predict with accuracy which patient will be able to tolerate the period of temporary ischemia. To extend this observation to recommend cardiorespiratory arrest in all patients with a complex MCA aneurysm cannot be supported by the data in the present series.

Since most postoperative deficits can be attributed to distal emboli, or to permanent branch occlusion, it is unlikely that complete cardiac arrest with extracorporeal circulation would offer any benefit in the management of complex MCA aneurysms, and would likely only increase the perioperative morbidity from the inherent risks of the cardioplegia and extracorporeal circulation. 


\section{References}

1) Allcock JM, Canham PB: Angiographic study of the growth of intracranial aneurysms. J Neurosurg 45: 617-621, 1976

2) Andrews RJ, Spiegel PK: Intracranial aneurysms. Age, sex, blood pressure, and multiplicity in an unselected series of patients. J Neurosurg 51: 27-32, 1979

3) Ausman JI, Charbel FT, Diaz FG, Malik GM, Dujovny M: Timing of surgery in grade IV aneurysmal subarachnoid hemorrhage. Surgical Forum 39: 499501, 1988

4) Ausman JI, Diaz FG, Malik GM, Andrews BT, McCormick P, Balakrishnam G: Management of cerebral aneurysms: Further facts and additional myths. Surg Neurol 32: 21-35, 1989

5) Ausman JI, Diaz FG, Sadasivan B, Gonzales-Portillo M, Malik GM, Deopujari CE: Giant intracranial aneurysm surgery: The role of microvascular reconstruction. Surg Neurol 34: 8-15, 1990

6) Cabezudo-Artero J, Ausman JI, Dujovny M, OrdonezMora E, Umansky F, Diaz FG, Mirchandani H, Berman SK: Middle cerebral artery reconstruction. Surg Neurol 24: 5-11, 1985

7) Charbel FT, Ausman JI, Diaz FG, Malik GM, Dujovny $\mathrm{M}$, Sanders J: Temporary clipping in aneurysm surgery: Technique and results. Surg Neurol 36: 83-90, 1991

8) Da Pian R, Pasqualin A, Scienza R: Direct microsurgical approach to aneurysms of the internal carotid bifurcation. Surg Neurol 13: 27-37, 1980

9) Dandy WE: Intracranial Arterial Aneurysms. Ithica, NY, Comstock Pub, 1944, pp 1-100

10) Diaz FG: Surgical treatment of aneurysms in the cavernous sinus. Contemp Neurosurg 10 (26): 1-6, 1989

11) Diaz FG, Umansky F, Mehta B, Montoya S, Dujovny M, Ausman JI, Cabezudo J: Cerebral revascularization to a main limb of the middle cerebral artery in the sylvian fissure. An alternative approach to conventional anastomosis. J Neurosurg 63: 21-29, 1985

12) Gibo H, Lenkey C, Rhoton AL: Microsurgical anatomy of the supraclinoid portion of the internal carotid artery. $J$ Neurosurg 55: 560-574, 1981

13) Gomes F, Dujovny M, Umansky F, Ausman JI, Diaz FG, Ray WJ, Mirchandani GF: Microsurgical anatomy of the recurrent artery of Heubner. J Neurosurg 60: 130-139, 1984

14) Gomes FB, Dujovny M, Umansky F, Berman SK, Diaz FG, Ausman JI, Mirchandani HG, Ray WJ: Microanatomy of the anterior cerebral artery. Surg Neurol 26: 129-141, 1986

15) Grand W: Microsurgical anatomy of the proximal middle cerebral artery and internal carotid bifurcation. Neurosurgery 7: 215-218, 1980

16) Higashida RT, Halback VV, Barnwell SL, Dowd CF, Dormandy B, Bell J, Hieshima GB: Treatment of intracranial aneurysms with preservation of the parent vessel. AJNR Am J Neuroradiol 11: 633-640, 1990
17) Hunt WE, Hess RM: Surgical risk as related to time of intervention in the repair of intracranial aneurysms. J Neurosurg 28: 14-20, 1968

18) Kassell NF, Sasaki T, Colohan AR, Nazar G: Cerebral vasospasm following aneurysmal subarachnoid hemorrhage. Stroke 16: 562-572, 1985

19) Kobayashi S, Sugita K, Nakagawa F: An approach to a basilar aneurysm above the bifurcation of the internal carotid artery. Case report. J Neurosurg 59: 10821084,1983

20) Locksley HB: Report on the Cooperative Study of Intracranial Aneurysms and Subarachnoid Hemorrhage. Section V, part 1. Natural history of subarachnoid hemorrhage, intracranial aneurysms and arteriovenous malformations. Based on 6368 cases in the Cooperative Study. J Neurosurg 25: 219239, 1966

21) Pool JL, Potts DG: Aneurysms and Arteriovenous Anomalies of the Brain: Diagnosis and Treatment. New York, Hoeber Medical Division, Harper \& Row, 1965

22) Silverberg GD, Reitz BA, Ream AK: Hypothermia and cardiac arrest in the treatment of giant aneurysms of the cerebral circulation and hemangioblastoma of the medulla. J Neurosurg 55: 337-346, 1981

23) Spetzger U, Reui J, Weis J, Bertalanfy H, Thron A, Gilsbach JM: Microsurgically produced bifurcation aneurysms in a rabbit model for endovascular coil embolization. J Neurosurg 85: 488-495, 1996

24) Suzuki J: Cerebral Aneurysms, Experience with 1000 Directly Operated Cases. Tokyo, Neuron Publishing, 1979, $100 \mathrm{pp}$

25) Umansky F, Gomes FB, Dujovny $M$, Diaz FG, Ausman JI, Mirchandani HG, Berman SK: The perforating branches of the middle cerebral artery: A microanatomical study. I Neurosurg 62: 261-268, 1985

26) Umansky F, Montoya Juarez S, Dujovny M, Ausman JI, Diaz FG, Gomes F, Mirchandani HG, Ray WJ: Microsurgical anatomy of the proximal segments of the middle cerebral artery. J Neurosurg 61: 458-467, 1984

27) Varkey GP (ed): Anesthetic Considerations in the Surgical Repair of Intracranial Aneurysms. International Anesthesiology Clinics. Boston, Little Brown, 1982, pp 75-150

28) Yasargil MG, Antic J, Laciga R, Jain KK, Hodosh RM, Smith RD: Microsurgical pterional approach to aneurysms of the basilar bifurcation. Surg Neurol 6: 83-91, 1976

29) Yokoh A, Ausman JI, Dujovny M, Diaz FG, Berman SK, Sanders J, Mirchandani HG: Anterior cerebral artery reconstruction. Neurosurgery 19: 26-35, 1986

Address reprint requests to: F. G. Diaz, M.D., Neurological Surgery Department, Wayne State University School of Medicine, 4201 St. Antoine Blvd., Detroit, Michigan, U.S.A. 